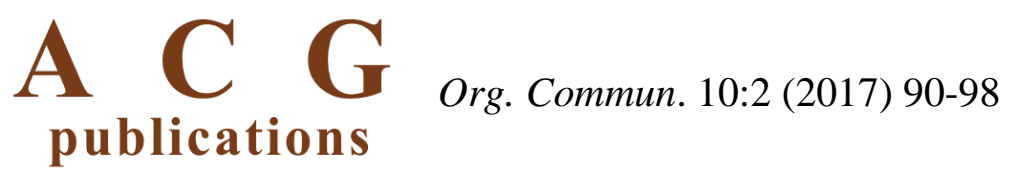

organic communications

\title{
Synthesis and anti-bacterial activity of novel 1,3-phenylene-bis-N- acetyl- and N-phenylpyrazole derivatives
}

\author{
Gökçe Demir, Meliha Burcu Gürdere, Mustafa Ceylan \\ and Yakup Budak ${ }^{*}$
}

\author{
Department of Chemistry, Faculty of Arts and Sciences, Gaziosmanpasa University, \\ 60250, Tokat, Türkiye
}

(Received December 14, 2016; Revised January 27, 2017; Accepted January 31, 2017)

\begin{abstract}
The 1,1'-(5,5'-(1,3-phenylene)bis(3-aryl-4,5-dihydro-1H-pyrazole-5,1-diyl))di-ethanone (5a-e) and 1,3bis(1-phenyl-3-(aryl)-4,5-dihydro-1H-pyrazol-5-yl)benzene (6a-e) were synthesized by addition of hydrazine hydrate and/or phenylhydrazine to 1,3-phenylene-bis-chalcone derivatives (3a-e), respectively. The structures of obtained compounds (5a-e and 6a-e) were characterized using the spectroscopic methods (NMR, IR) and elemental analysis. Addition, the in vitro antibacterial activities of compounds (5a-e) was tested against the five human pathogenic bacteria. Gentamycin and Fluconazole were used as positive control. The results were given as inhibition zone ( $\mathrm{mm}$ ) and the compounds $\mathbf{5 d}$ and $\mathbf{5 e}$ showed the same activity with standard (Fluconazole) against C. albinas.
\end{abstract}

Keywords: 1,3-Phenylene-bis-N-acetylpyrazoles; 1,3-phenylene-bis-N-phenylpyrazoles; antibacterial activity. (C) 2017 ACG Publications. All rights reserved.

\section{Introduction}

Pyrazole ring is well known for their wide range of biological activities such as analgesic, antiinflammatory, antipyretic, antiarrhythmic, sedative, muscle relaxant, psychoanaleptic, monoamine oxidase inhibitor, anti-diabetic and antimicrobial properties. ${ }^{1-8}$ Addition, pyrazole derivatives play an important role in the development of pesticides and medicines. Several of pyrazole products are available and widely used as fungicides, antiviral agents, analgesic agents, insecticides and herbicides. ${ }^{9-}$ ${ }^{11}$ For thus, pyrazole derivatives have attracted considerable attention in the recent years for their diverse biological activities. ${ }^{12-17}$

In this paper, the novel 1,1'-(5,5'-(1,3-phenylene)bis(3-aryl-4,5-dihydro-1H-pyrazole-5,1diyl))diethanone (5a-e) and 1,3-bis(1-phenyl-3-(aryl)-4,5-dihydro-1H-pyrazol-5-yl)benzene (6a-e) were synthesized in good yields and evaluated the antibacterial activities of compounds (5a-e) against some human pathogenic bacteria.

\section{Experimental}

IR spectrums $(\mathrm{KBr})$ were recorded on a Jasco FT/IR-430 spectrometer. ${ }^{1} \mathrm{H}$ and ${ }^{13} \mathrm{C}$ NMR spectra were recorded on a Bruker Avance DPX-400 instrument. As internal $\delta(0.00)$ for standards served TMS

\footnotetext{
* Corresponding author: E-Mail: yakup.budak@gop.edu.tr, Phone: + 90 3562521616; Fax: + 903562521585.
} 
${ }^{1} \mathrm{H}$ NMR and $\mathrm{CDCl}_{3} \delta(77.0)$ for ${ }^{13} \mathrm{C}$ NMR spectra, $J$ values are given in Hz. Melting points were measured on Electrothermal 9100 apparatus. Elemental analyses were obtained from a LECO CHNS 932 Elemental Analyzer. The major chemicals were purchased from Sigma-Aldrich and Fluka.

2.1 General Procedure for the Synthesis of Bis-Chalcone Derivatives (3a-e): ${ }^{18-22}$ Aril ketones derivatives $(\mathbf{1 a - h})(1 \mathrm{mmol})$ in $\mathrm{EtOH}(20 \mathrm{ml})$ were added to $\mathrm{NaOH}(2.5 \mathrm{M}, 6 \mathrm{ml})$. The mixture was stirred for $3 \mathrm{~min}$ and was added (2) $(1 \mathrm{mmol})$ and was stirred for $4 \mathrm{~h}$ at r.t. Start mixing a few minutes after it was observed that the collapse occurred. By reaction the precipitate formed was filtered off. After washing with ethanol several times with methylene chloride / hexane (3/7) mixture was crystallized.

(2E,2'E)-3,3'-(1,3-phenylene)bis(1-phenylprop-2-en-1-one) (3a):Viscous oil. Yield: 73\%. IR KBr $\left(\mathrm{cm}^{-1}\right): 3058,3035,2805,1664,1600,1575,1438,1334,1276,1214,1172,1031,1016,993,975,857$, 767, 682. ${ }^{1} \mathrm{H}$ NMR $\left(\delta\right.$, ppm, $\left.400 \mathrm{MHz}, \mathrm{CDCl}_{3}\right): 8.07(\mathrm{~d}, J=7.6 \mathrm{~Hz}, 4 \mathrm{H}), 7.90(\mathrm{~s}, 1 \mathrm{H}), 7.86(\mathrm{~d}, J=15.6$ $\mathrm{Hz}, 2 \mathrm{H}), 7.32(\mathrm{~d}, J=7.6 \mathrm{~Hz}, 2 \mathrm{H}), 7.65-7.50(\mathrm{~m}, 9 \mathrm{H}) ;{ }^{13} \mathrm{C} \mathrm{NMR}\left(\delta, \mathrm{ppm}, 100 \mathrm{MHz}, \mathrm{CDCl}_{3}\right): 190.3(2 \mathrm{C})$, 143.8 (2C), 138.0 (2C), 135.7 (2C), 132.9 (2C), 130.1 (2C), 129.6, 128.7 (4C), 128.6 (4C), 128.3, 123.0 (2C). Anal. calc. for $\mathrm{C}_{24} \mathrm{H}_{18} \mathrm{O}_{2}$ : C, 85.18; H, 5.36. Found: C, 85.08; H, 5.28.

(2E,2'E)-3,3'-(1,3-phenylene)bis[1-(4-methylphenyl)prop-2-en-1-one] (3b):Colorless solid. M.p. $191{ }^{\circ} \mathrm{C}$. Yield: $87 \%$. IR $\mathrm{KBr}\left(\mathrm{cm}^{-1}\right): 3023,2919,1654,1604,1334,1270,1203,1168,1029,784 .{ }^{1} \mathrm{H}$ NMR ( $\delta$, ppm, $\left.400 \mathrm{MHz}, \mathrm{CDCl}_{3}\right): 7.99(d, J=7.2 \mathrm{~Hz}, 4 \mathrm{H}), 7.90(\mathrm{~s}, 1 \mathrm{H}), 7.85(d, J=16.0 \mathrm{~Hz}, 2 \mathrm{H}), 7.71$ $(\mathrm{d}, J=7.6 \mathrm{~Hz}, 2 \mathrm{H}), 7.61(d, J=16.0 \mathrm{~Hz}, 2 \mathrm{H}), 7.52(\mathrm{t}, J=7.6 \mathrm{~Hz}, 1 \mathrm{H}), 7.35(\mathrm{~d}, J=7.6 \mathrm{~Hz}, 4 \mathrm{H}), 2.47(\mathrm{~s}$, $6 \mathrm{H}) ;{ }^{13} \mathrm{C}$ NMR $\left(\delta, \mathrm{ppm}, 100 \mathrm{MHz}, \mathrm{CDCl}_{3}\right.$ ): 189.8 (2C), 143.8, 143.4 (3C), 135.8, 135.5, 129.9 (3C), 129.6, 129.4 (4C), 128.7 (4C), 128.2, 123.0 (3C), 21.7 (2C). Anal. calc. for $\mathrm{C}_{26} \mathrm{H}_{22} \mathrm{O}_{2}: \mathrm{C}, 85.22 ; \mathrm{H}$, 6.05. Found: C, 85.16; H, 5.98.

(2E,2'E)-3,3'-(1,3-phenylene)bis[1-(4-methoxyphenyl)prop-2-en-1-one] (3c): Colorless solid. M.p. $181{ }^{\circ}$ C. Yield: $98 \%$. IR $\mathrm{KBr}\left(\mathrm{cm}^{-1}\right)$ : 3064, 3002, 2933, 2838, 1650, 1596, 1509, 1450, 1415, 1324, $1257,1211,1176,1118,1022,835,796,572 .{ }^{1} \mathrm{H}$ NMR $\left(\delta, \mathrm{ppm}, 400 \mathrm{MHz}, \mathrm{CDCl}_{3}\right): 8.08(d, J=8.8 \mathrm{~Hz}$, $4 \mathrm{H}), 7.92(\mathrm{~s}, 1 \mathrm{H}), 7.84(d, J=15.6 \mathrm{~Hz}, 2 \mathrm{H}), 7.70(\mathrm{~d}, J=7.6 \mathrm{~Hz}, 2 \mathrm{H}), 7.62(d, J=15.6 \mathrm{~Hz}, 2 \mathrm{H}),, 7.50(\mathrm{t}$, $J=7.6 \mathrm{~Hz}, 1 \mathrm{H}), 7.02(\mathrm{~d}, J=8.8 \mathrm{~Hz}, 4 \mathrm{H}), 3.83(\mathrm{~s}, 6 \mathrm{H},) ;{ }^{13} \mathrm{C}$ NMR $\left(\delta, \mathrm{ppm}, 100 \mathrm{MHz}, \mathrm{CDCl}_{3}\right): 188.5$ (2C), 163.6 (2C), 143.0 (3C), 135.8, 130.9 (4C), 129.8 (3C), 129.5, 128.1, 122.8 (3C), 113.9 (4C), 55.5 (2C). Anal. calc. for $\mathrm{C}_{26} \mathrm{H}_{22} \mathrm{O}_{4}$ : C, 78.37; H, 5.57. Found: C, 78.26; H, 5.48.

(2E,2'E)-3,3'-(1,3-phenylene)bis[1-(4-bromophenyl)prop-2-en-1-one] (3d): Colorless solid. M.p. $183{ }^{\circ} \mathrm{C}$. Yield: $97 \%$. IR KBr $\left(\mathrm{cm}^{-1}\right): 3083,3060,2987,1654,1606,1583,1330,1280,1209,1070,1006$, 973, 806. ${ }^{1} \mathrm{H}$ NMR $\left(\delta\right.$, ppm, $\left.400 \mathrm{MHz}, \mathrm{CDCl}_{3}\right)$ : 7.95-7.84 (m, 6H), 7.87-7.77 (m, 6H), 7.61-7.44 (m 4H). ${ }^{13} \mathrm{C}$ NMR $\left(\delta, p p m, 100 \mathrm{MHz}, \mathrm{CDCl}_{3}\right): 188.5$ (2C), 144.3 (3C), 136.9 (2C), 135.7 (3C), 132.4 (4C), 131.6, 131.1 (4C), 130.0, 127.9, 123.1 (3C). Anal. calc. for $\mathrm{C}_{24} \mathrm{H}_{16} \mathrm{Br}_{2} \mathrm{O}_{2}$ : C, 58.09; H, 3.25. Found: C, $58.01 ; \mathrm{H}, 3.19$.

(2E,2'E)-3,3'-(1,3-phenylene)bis[1-(4-chlorophenyl)prop-2-en-1-one] (3e): Colorless solid. M.p. $201{ }^{\circ} \mathrm{C}$. Yield: $98 \%$. IR KBr $\left(\mathrm{cm}^{-1}\right): 3062,3018,2884,1656,1600,1484,1398,1336,1309,1205,1166$, $1083,1031,1008,788 .{ }^{1} \mathrm{H}$ NMR $\left(\delta, \mathrm{ppm}, 400 \mathrm{MHz}, \mathrm{CDCl}_{3}\right): 8.31(\mathrm{~s}, 1 \mathrm{H}), 8.21(\mathrm{~d}, J=8.8 \mathrm{~Hz} 4 \mathrm{H}), 8.05$ $(\mathrm{d}, J=16.0 \mathrm{~Hz}, 2 \mathrm{H}), 7.99(\mathrm{~d}, J=8.0 \mathrm{~Hz}, 2 \mathrm{H}), 7.82(\mathrm{~d}, J=16.0 \mathrm{~Hz}, 2 \mathrm{H}), 7.67(\mathrm{~d}, J=8.8 \mathrm{~Hz}, 4 \mathrm{H}), 7.57$ $(\mathrm{t}, J=7.6 \mathrm{~Hz}, 1 \mathrm{H}) .{ }^{13} \mathrm{C} \mathrm{NMR}\left(\delta, \mathrm{ppm}, 100 \mathrm{MHz}, \mathrm{CDCl}_{3}\right): 188.6(2 \mathrm{C}), 144.3(3 \mathrm{C}), 138.7(2 \mathrm{C}), 136.6$ (2C), 135.7 (2C), 131.5, 130.9 (4C), 129.9, 129.6, 129.4 (4C), 123.0 (2C). Anal. calc. for $\mathrm{C}_{24} \mathrm{H}_{16} \mathrm{Cl}_{2} \mathrm{O}_{2}$ : C, 70.77; H, 3.96. Found: C, 70.69; H, 3.88 . 
2.2 General Method for the Synthesis of 1,4-Phenylene-bis-N-acetylpyrazole (5a-h) and 1,4Phenylene-bis-N-phenylpyrazole Derivatives (7a-h): $:^{23-27}$

Bis-chalcone (1a-h) $(1 \mathrm{mmol})$ and hydrazine hydrate $(4 \mathrm{mmol})$ in acetic acid and/or phenylhydrazine $(4 \mathrm{mmol})$ in acetic acid/ethanol mixture were refluxed for 4 and 8 hours, respectively. The reaction mixture was poured into ice-bath, at the end of the reaction and was checked with $\mathrm{pH}$ paper to ensure neutralization of the medium by addition of ammonia. It was observed that the collapse occurs in the mixture to stand overnight and the precipitate formed was filtered off, was allowed to dry. The resulting material was obtained as a pure.

5,5'-(1,3-phenylene)bis(1-acetyl-3-phenyl-4,5-dihydro-1H-pyrazole) (5a):Colorless solid. M.p. $263{ }^{\circ} \mathrm{C}$. Yield: 89\%. IR KBr $\left(\mathrm{cm}^{-1}\right)$ : 3062, 3025, 2927, 2836, 1650, 1411, 1355, 1324, 1147, 898, 765, 696. ${ }^{1} \mathrm{H}$ NMR $\left(\delta, \mathrm{ppm}, 400 \mathrm{MHz}, \mathrm{CDCl}_{3}\right)$ : 7.70-7.65 (m, 4H), 7.45-7.44 (m, 6H), 7.30-7.25 (m, 2H), $7.13(\mathrm{~d}, J=8.0 \mathrm{~Hz}, 1 \mathrm{H}), 7.06(\mathrm{t}, J=8.0 \mathrm{~Hz}, 1 \mathrm{H}), 5.61-5.54(\mathrm{~m}, 2 \mathrm{H}), 3.78-3.71(\mathrm{~m}, 2 \mathrm{H}), 3.25-3.17(\mathrm{~m}$, $2 \mathrm{H}), 2.46(\mathrm{~s}, 3 \mathrm{H}), 2.37$ (s, 3H). ${ }^{13} \mathrm{C}$ NMR $\left(\delta, \mathrm{ppm}, 100 \mathrm{MHz}, \mathrm{CDCl}_{3}\right): 169.8,168.8,154.4,154.3,142.5$, $142.3,140.7,129.7$ (4C), 128.8, 128.5, 126.6 (4C), 126.4, 124.9, 124.4, 124.0, 122.8, 59.9, 59.8, 42.4 (2C), 21.9, 21.5. Anal. calc. for $\mathrm{C}_{28} \mathrm{H}_{26} \mathrm{~N}_{4} \mathrm{O}_{2}$ : C, 74.65; H, 5.82; N, 12.44. Found: C, 74.59; H, 5.78; N, 12.33 .

5,5'-(1,3-phenylene)bis[1-acetyl-3-(4-methylphenyl)-4,5-dihydro-1H-pyrazole] (5b): Viscous oil. Yield: $96 \%$. IR $\mathrm{KBr}\left(\mathrm{cm}^{-1}\right): 3029,2919,2854,1658,1427,1359,1324,1263,1178,1135,1029$, 952, 862, 734, 626. ${ }^{1} \mathrm{H}$ NMR $\left(\delta, \mathrm{ppm}, 400 \mathrm{MHz}, \mathrm{CDCl}_{3}\right): 7.64(\mathrm{~d}, J=7.6 \mathrm{~Hz}, 4 \mathrm{H}), 7.28-7.23(\mathrm{~m}, 6 \mathrm{H})$, $7.12(\mathrm{~d}, J=8.0 \mathrm{~Hz}, 2 \mathrm{H}), 5.59-5.51(\mathrm{~m}, 2 \mathrm{H}), 3.74-3.66(\mathrm{~m}, 2 \mathrm{H}), 3.21-3.14(\mathrm{~m}, 2 \mathrm{H}), 2.44(\mathrm{~s}, 3 \mathrm{H}), 2.41(\mathrm{~s}$, $6 \mathrm{H}) .2 .39(\mathrm{~s}, 3 \mathrm{H}) ;{ }^{13} \mathrm{C}$ NMR $\left(\delta, \mathrm{ppm}, 100 \mathrm{MHz}, \mathrm{CDCl}_{3}\right): 168.9,168.7,154.4,154.3,142.5,142.3,140.7$, $129.9,129.7$ (4C), 128.5, 128.3, 126.6 (4C), 124.9, 124.5, 124.1, 122.8, 59.9, 59.8, 42.4 (2C), 21.9, 21.8, 21.5 (2C). $\mathrm{C}_{30} \mathrm{H}_{30} \mathrm{Br}_{2} \mathrm{~N}_{4} \mathrm{O}_{2}$ : C, 75.29; H, 6.32; N, 11.71. Found: $\mathrm{C}, 75.14 ; \mathrm{H}, 6.26 ; \mathrm{N}, 11.65$.

5,5'-(1,3-phenylene)bis[1-acetyl-3-(4-methoxyphenyl)-4,5-dihydro-1H-pyrazole] (5c): Viscous oil. Yield: 73\%. IR KBr $\left(\mathrm{cm}^{-1}\right)$ : 3056, 3002, 2953, 2884, 1664, 1604, 1511, 1425, 1359, 1328, 1253, 1172, 1035, 831, 700. ${ }^{1} \mathrm{H}$ NMR ( $\delta$, ppm, $\left.400 \mathrm{MHz}, \mathrm{CDCl}_{3}\right): 7.68$ (d, $\left.J=8.4 \mathrm{~Hz}, 4 \mathrm{H}\right), 7.27(\mathrm{~s}, 1 \mathrm{H}), 7.24$ $(\mathrm{d}, J=7.6 \mathrm{~Hz}, 1 \mathrm{H}), 7.12(\mathrm{t}, J=7.6 \mathrm{~Hz}, 1 \mathrm{H}), 7.05(\mathrm{~d}, J=7.6 \mathrm{~Hz}, 1 \mathrm{H}), 6.94(\mathrm{~d}, J=8.4 \mathrm{~Hz}, 4 \mathrm{H}), 5.55(\mathrm{t}, J$ $=12.0 \mathrm{~Hz}, 2 \mathrm{H}), 3.88(\mathrm{~s}, 6 \mathrm{H}), 3.73-3.65(\mathrm{~m}, 2 \mathrm{H}), 3.19-3.12(\mathrm{~m}, 2 \mathrm{H}), 2.43(\mathrm{~s}, 3 \mathrm{H}), 2.37(\mathrm{~s}, 3 \mathrm{H}) .{ }^{13} \mathrm{C} \mathrm{NMR}$ $\left(\delta, \mathrm{ppm}, 100 \mathrm{MHz}, \mathrm{CDCl}_{3}\right)$ : 168.8, 168.7, 161.4, 154.0, 153.9, 142.6, 142.4, 129.8, 129.4, 128.3, 128.2 (2C), 124.9, 124.0, 123.9, 123.8 (2C), 122.8, 114.1 (4C), 59.8, 59.7, 55.4 (2C), 42.5 (2C), 22.0, 21.9. $\mathrm{C}_{30} \mathrm{H}_{30} \mathrm{Br}_{2} \mathrm{~N}_{4} \mathrm{O}_{4}: \mathrm{C}, 70.57 ; \mathrm{H}, 5.92 ; \mathrm{N}, 10.97$. Found: C, 70.49; H, 5.88; N, 10.83 .

5,5'-(1,3-phenylene)bis[1-acetyl-3-(4-bromophenyl)-4,5-dihydro-1H-pyrazole] (5d): Colorless solid. M.p. $180{ }^{\circ}$ C. Yield: $92 \%$. IR KBr $\left(\mathrm{cm}^{-1}\right)$ : 3052, 2979, 2927, 1658, 1592, 1409, 1361, 1319, 1263 , 1251, 1139, 1070, 1012, 952, 819, 734, 532. ${ }^{1} \mathrm{H}$ NMR $\left(\delta, \mathrm{ppm}, 400 \mathrm{MHz}, \mathrm{CDCl}_{3}\right): 7.61(\mathrm{~d}, J=8.0 \mathrm{~Hz}$, $4 \mathrm{H}), 7.57(\mathrm{~d}, J=8.0 \mathrm{~Hz}, 4 \mathrm{H}), 7.29-7.27(\mathrm{~m}, 2 \mathrm{H}), 7.12(\mathrm{~d}, J=8.0 \mathrm{~Hz}, 1 \mathrm{H}), 7.06(\mathrm{t}, J=8.0 \mathrm{~Hz}, 1 \mathrm{H}), 5.61-$ $5.54(\mathrm{~m}, 2 \mathrm{H}), 3.76-3.65(\mathrm{~m}, 2 \mathrm{H}), 3.20-3.13(\mathrm{~m}, 2 \mathrm{H}), 2.43(\mathrm{~s}, 3 \mathrm{H}), 2.38(\mathrm{~s}, 3 \mathrm{H}) ;{ }^{13} \mathrm{C}$ NMR $(\delta, \mathrm{ppm}, 100$ $\mathrm{MHz}, \mathrm{CDCl}_{3}$ ): 169.0 (2C), 153.2 (2C), 142.3, 142.1, 131.9 (4C), 130.2, 129.6, 128.1 (4C), 124.9 (2C), 124.7 (2C), 124.1, 122.6, 60.1 (2C), 42.2 (2C), 22.0, 21.9. Anal. calc. for $\mathrm{C}_{28} \mathrm{H}_{24} \mathrm{Br}_{2} \mathrm{~N}_{4} \mathrm{O}_{2}: \mathrm{C}, 55.28 ; \mathrm{H}$, $3.98 ;$ N, 9.21. Found: C, 55.20; H, 3.89; N, 9.18.

5,5'-(1,3-phenylene)bis[1-acetyl-3-(4-chlorophenyl)-4,5-dihydro-1H-pyrazole] (5e): Colorless solid. M.p. $194{ }^{\circ} \mathrm{C}$. Yield: 66\%. IR KBr $\left(\mathrm{cm}^{-1}\right)$ : 3056, 2927, 1664, 1594, 1486, 1419, 1400, 1361, 1322, 1095, 1006, 827, 734. ${ }^{1} \mathrm{H}$ NMR ( $\delta$, ppm, $\left.400 \mathrm{MHz}, \mathrm{CDCl}_{3}\right): 7.67(\mathrm{~d}, J=8.0 \mathrm{~Hz}, 4 \mathrm{H}), 7.41(\mathrm{~d}, J=8.0$ $\mathrm{Hz}, 4 \mathrm{H}), 7.31-7.25(\mathrm{~m}, 2 \mathrm{H}), 7.12(\mathrm{~d}, J=8.0 \mathrm{~Hz}, 1 \mathrm{H}), 7.06(\mathrm{t}, J=8.0 \mathrm{~Hz}, 1 \mathrm{H}), 5.61-5.54(\mathrm{~m}, 2 \mathrm{H}), 3.76-$ $3.67(\mathrm{~m}, 2 \mathrm{H}), 3.20-3.13(\mathrm{~m}, 2 \mathrm{H}), 2.44(\mathrm{~s}, 3 \mathrm{H}), 2.38(\mathrm{~s}, 3 \mathrm{H}) .{ }^{13} \mathrm{C}$ NMR $\left(\delta, \mathrm{ppm}, 100 \mathrm{MHz}, \mathrm{CDCl}_{3}\right)$ : 169.0, 168.9, 153.1, 153.0, 142.3, 142.1, 136.3 (2C), 129.7, 129.5, 129.2 (4C), 127.8 (4C), 126.8, 124.9, 
124.5, 124.0, 60.1, 60.0, 42.3, 42.2, 22.0, 21.9. Anal. calc. for $\mathrm{C}_{28} \mathrm{H}_{24} \mathrm{Cl}_{2} \mathrm{~N}_{4} \mathrm{O}_{2}: \mathrm{C}, 64.74 ; \mathrm{H}, 4.66 ; \mathrm{N}$, 10.79. Found: C, 64.66; H, 4.57; N, 10.69 .

1,3-bis(1,3-diphenyl-4,5-dihydro-1H-pyrazol-5-yl)benzene (7a): Yellow solid. M.p. $158{ }^{\circ} \mathrm{C}$. Yield: 89\%. IR $\mathrm{KBr}\left(\mathrm{cm}^{-1}\right): 3076,3012,2928,2843,1409,1386,1361,1145,887,745,686 .{ }^{1} \mathrm{H}$ NMR $(\delta$, ppm, $\left.400 \mathrm{MHz}, \mathrm{CDCl}_{3}\right): 7.70(\mathrm{~d}, J=7.6 \mathrm{~Hz}, 4 \mathrm{H}), 7.48-7.36(\mathrm{~m}, 8 \mathrm{H}), 7.33-7.14(\mathrm{~m}, 8 \mathrm{H}), 7.00(\mathrm{~d}, J=8.0 \mathrm{~Hz}$, $2 \mathrm{H}), 6.81(\mathrm{~d}, J=7.6 \mathrm{~Hz}, 2 \mathrm{H}), 5.17-5.12(\mathrm{~m}, 2 \mathrm{H}), 3.80-3.73(\mathrm{~m}, 2 \mathrm{H}), 3.01-2.94(\mathrm{~m}, 2 \mathrm{H}) .{ }^{13} \mathrm{C}$ NMR $(\delta$, ppm, $100 \mathrm{MHz}, \mathrm{CDCl}_{3}$ ): 147.1, 146.9, 144.8, 143.6 (2C), 138.7, 130.0, 129.8 (4C), 129.3 (4C), 128.8 (4C), 125.7 (4C), 125.2, 125.1, 123.9, 119.0 (2C), 113.4 (4C), 64.4 (2C), 43.6, 43.5. Anal. calc. for $\mathrm{C}_{36} \mathrm{H}_{30} \mathrm{~N}_{4}: \mathrm{C}, 83.37 ; \mathrm{H}, 5.83 ; \mathrm{N}, 10.80$. Found: C, 83.29; H, 5.74; N, 10.71.

1,3-bis(1-phenyl-3-(p-tolyl)-4,5-dihydro-1H-pyrazol-5-yl)benzene (7b):Yellow solid. M.p. 189 ${ }^{\circ}$ C. Yield: $96 \%$. IR KBr $\left(\mathrm{cm}^{-1}\right)$ : 3016, 2901, 2845, 1433, 1341, 1319, 1262, 1169, 1133, 1001, 945, 853, 725, 616. ${ }^{1} \mathrm{H}$ NMR $\left(\delta, \mathrm{ppm}, 400 \mathrm{MHz}, \mathrm{CDCl}_{3}\right): 7.64(\mathrm{~d}, J=7.6 \mathrm{~Hz}, 4 \mathrm{H}), 7.31(\mathrm{~d}, J=7.6 \mathrm{~Hz}, 4 \mathrm{H}), 7.22-$ $7.13(\mathrm{~m}, 8 \mathrm{H}), 7.06(\mathrm{~d}, J=8.0 \mathrm{~Hz}, 2 \mathrm{H}), 6.99(\mathrm{~d}, J=8.0 \mathrm{~Hz}, 2 \mathrm{H}) .6 .08(\mathrm{t}, J=7.6 \mathrm{~Hz}, 2 \mathrm{H}), 5.22-5.18(\mathrm{~m}$, $2 \mathrm{H}), 3.86-3.77(\mathrm{~m}, 2 \mathrm{H}), 3.17-3.10(\mathrm{~m}, 2 \mathrm{H}), 2.38(\mathrm{~s}, 6 \mathrm{H}) .{ }^{13} \mathrm{C} \mathrm{NMR}\left(\delta, \mathrm{ppm}, 100 \mathrm{MHz}, \mathrm{CDCl}_{3}\right): 147.1$, 146.9, 144.8, 143.6 (2C), 138.7, 130.0, 129.8 (4C), 129.3 (4C), 128.8 (4C), 125.7 (3C), 125.2, 125.1, 123.9, 119.0 (2C), $113.4(3 \mathrm{C}), 64.4(2 \mathrm{C}), 43.6,43.5,21.4(2 \mathrm{C})$. Anal. calc. for $\mathrm{C}_{38} \mathrm{H}_{34} \mathrm{~N}_{4}: \mathrm{C}, 83.48 ; \mathrm{H}$, 6.27 ; N, 10.25. Found: C, 83.33; H, 6.18; N, 10.23.

1,3-bis(3-(4-methoxyphenyl)-1-phenyl-4,5-dihydro-1H-pyrazol-5-yl)benzene (7c): Yellow solid. M.p. $199{ }^{\circ} \mathrm{C}$. Yield: 88\%. IR KBr $\left(\mathrm{cm}^{-1}\right)$ : 3054, 3016, 2949, 2876, 1529, 1442, 1335, 1309, 1243, 1190 , 1023, 844, 719. ${ }^{1} \mathrm{H}$ NMR $\left(\delta, \mathrm{ppm}, 400 \mathrm{MHz}, \mathrm{CDCl}_{3}\right): 7.68(\mathrm{~d}, J=8.4 \mathrm{~Hz}, 4 \mathrm{H}), 7.33-7.13(\mathrm{~m}, 8 \mathrm{H}), 7.05$ $(\mathrm{d}, J=8.0 \mathrm{~Hz}, 2 \mathrm{H}), 6.98(\mathrm{~d}, J=8.0 \mathrm{~Hz}, 2 \mathrm{H}), 6.94(\mathrm{~d}, J=8.4 \mathrm{~Hz}, 4 \mathrm{H}), 6.79(\mathrm{t}, J=7.2 \mathrm{~Hz}, 2 \mathrm{H}), 5.23-5.16$ $(\mathrm{m}, 2 \mathrm{H}), 3.87(\mathrm{~s}, 6 \mathrm{H}), 3.83-3.76(\mathrm{~m}, 2 \mathrm{H}), 3.16-3.05(\mathrm{~m}, 2 \mathrm{H}) .{ }^{13} \mathrm{C} \mathrm{NMR}\left(\delta, \mathrm{ppm}, 100 \mathrm{MHz}, \mathrm{CDCl}_{3}\right)$ : 160.2, 160.1, 147.0, 146.8, 145.3, 145.1, $143.6(2 \mathrm{H}), 130.2,130.0,128.7(4 \mathrm{H}), 127.3(2 \mathrm{H}), 127.2(2 \mathrm{H})$, 125.5, 125.4, 125.2, 125.1, 123.9, 123.8, $118.9(2 \mathrm{H}), 114.0(4 \mathrm{C}), 113.3(2 \mathrm{H}), 64.5(2 \mathrm{C}), 55.4(2 \mathrm{C}), 43.8$, 43.7. Anal. calc. for $\mathrm{C}_{38} \mathrm{H}_{34} \mathrm{~N}_{4} \mathrm{O}_{2}$ : C, 78.87; H, 5.92; N, 9.68. Found: C, 78.78; H, 5.87; N, 9.59.

1,3-bis(3-(4-bromophenyl)-1-phenyl-4,5-dihydro-1H-pyrazol-5-yl)benzene (7d): Yellow solid. M.p. $193{ }^{\circ}$ C. Yield: 63\%. IR KBr $\left(\mathrm{cm}^{-1}\right): 3043,2967,2935,1589,1459,1348,1301,1258,1246,1142$, $1068,1009,963,823,748,526 .{ }^{1} \mathrm{H}$ NMR $\left(\delta, \mathrm{ppm}, 400 \mathrm{MHz}, \mathrm{CDCl}_{3}\right): 7.58(\mathrm{~d}, J=8.4 \mathrm{~Hz}, 4 \mathrm{H}), 7.52(\mathrm{~d}$, $J=8.4 \mathrm{~Hz}, 4 \mathrm{H}), 7.24-7.12(\mathrm{~m}, 8 \mathrm{H}), 7.04(\mathrm{~d}, J=8.0 \mathrm{~Hz}, 2 \mathrm{H}), 6.97(\mathrm{~d}, J=7.6 \mathrm{~Hz}, 2 \mathrm{H}), 6.84-6.79(\mathrm{~m}$, $2 \mathrm{H})$, 5.29-5.22 (m, 2H), 3.85-3.75 (m, $2 \mathrm{H}), 3.14-3.05(\mathrm{~m}, 2 \mathrm{H}) .{ }^{13} \mathrm{C}$ NMR $\left(\delta\right.$, ppm, $\left.100 \mathrm{MHz}, \mathrm{CDCl}_{3}\right)$ : 145.8, 145.6, 144.6, 144.4, $143.3(2 \mathrm{H}), 133.8,131.7(2 \mathrm{H}), 131.6(2 \mathrm{H}), 131.5(2 \mathrm{H}), 128.9(6 \mathrm{H}), 127.2$ $(2 \mathrm{H}), 125.3(2 \mathrm{H}), 125.2,123.8,122.6(2 \mathrm{H}), 122.3,119.5,119.4,113.6,113.5,64.5(2 \mathrm{H}), 43.3,43.2$. Anal. calc. for $\mathrm{C}_{36} \mathrm{H}_{28} \mathrm{Br}_{2} \mathrm{~N}_{4}$ : C, 63.92; H, 4.17; N, 8.28. Found: C, 63.86; H, 4.09; N, 8.16.

1,3-bis(3-(4-chlorophenyl)-1-phenyl-4,5-dihydro-1H-pyrazol-5-yl)benzene (7e): Yellow solid. M.p. $204{ }^{\circ} \mathrm{C}$. Yield: 65\%. IR $\mathrm{KBr}\left(\mathrm{cm}^{-1}\right)$ : 3052, 2917, 1593, 1482, 1405, 1400, 1356, 1318, 1090, 1004, 834, 726. ${ }^{1} \mathrm{H}$ NMR $\left(\delta\right.$, ppm, $\left.400 \mathrm{MHz}, \mathrm{CDCl}_{3}\right): 7.65(\mathrm{~d}, J=8.4 \mathrm{~Hz}, 4 \mathrm{H}), 7.37(\mathrm{~d}, J=8.4 \mathrm{~Hz}, 4 \mathrm{H}), 7.26-$ $7.13(\mathrm{~m}, 8 \mathrm{H}), 7.04(\mathrm{~d}, J=8.0 \mathrm{~Hz}, 2 \mathrm{H}), 6.97(\mathrm{~d}, J=8.4 \mathrm{~Hz}, 2 \mathrm{H}), 6.85-6.79(\mathrm{~m}, 2 \mathrm{H}), 5.30-5.22(\mathrm{~m}, 2 \mathrm{H})$, 3.85-3.74 (m, 2H), 314-3.03 (m, 2H). ${ }^{13} \mathrm{C}$ NMR $\left(\delta, \mathrm{ppm}, 100 \mathrm{MHz}, \mathrm{CDCl}_{3}\right): 145.8,145.5,144.6,144.4$, $143.2(2 \mathrm{H}), 134.4,134.2,131.2,131.1,130.4,130.2,128.9(4 \mathrm{H}), 128.8(4 \mathrm{H}), 127.0(2 \mathrm{H}), 126.9(2 \mathrm{H})$, $125.3,125.2,123.8,123.6,119.5,119.4,113.6,113.5,64.5(2 \mathrm{H}), 43.4,43.2$. Anal. calc. for $\mathrm{C}_{36} \mathrm{H}_{28} \mathrm{Cl}_{2} \mathrm{~N}_{4}: \mathrm{C}, 73.59 ; \mathrm{H}, 4.80 ; \mathrm{N}, 9.54$. Found: C, 73.44; H, 4.71; N, 9.48.

\subsection{Antimicrobial activity}

The C. albicans was grown by $24 \mathrm{~h}$ of incubation at $25^{\circ} \mathrm{C}$ in Sabouraud Dextrose Broth (Merck) and the other bacteria were grown by $24 \mathrm{~h}$ of incubation at $36{ }^{\circ} \mathrm{C}$ in Mueller-Hinton Broth (Merck). Antibacterial activities were determined by disc-diffusion method ${ }^{28,29}$ using $100 \mu \mathrm{L}$ of suspension 
containing $10^{8} \mathrm{CFU} / \mathrm{mL}$ of bacteria and $10^{6} \mathrm{CFU} / \mathrm{mL}$ of yeast spread on Nutrient Agar (NA) and Sabouraud Dextrose Agar (SDA) medium, respectively. Starting dilution $(1 / 10 \mathrm{mg} / \mathrm{ml})$ was prepared by dissolving each of the compounds in DMSO. The blank discs (Oxoid $=6 \mathrm{~mm}$ in diameter) were impregnated with $20 \mu \mathrm{L}$ of each substance and placed on the inoculated agar. Gentamycin and Fluconazole were used as standard to determine the sensitivity of a strain of each microbial species tested. The inoculated plates were incubated at $37^{\circ} \mathrm{C}$ for 18 hours.

\section{Results and Discussion}

\subsection{Chemistry}

The starting materials 3a-e, 1,3-phenylene-bis-chalcone, were synthesized of well known Claisen-Schmidt condensation from the reaction of isophthaldehyde (1) with related ketones (2a-e) in the presence of $\mathrm{NaOH}$ in $\mathrm{EtOH}$ at r.t. for 4 hours (Scheme1, Table 1). According to our literature surveys, compound $\mathbf{3 a}$ is known the others are unknown. ${ }^{18}$ The structures of compounds were explained on the basis of spectral data (NMR, IR) and elemental analysis.

Then, the novel 1,3-phenylene-bis-N-acetyl- (5a-e), 1,1'-(5,5'-(1,3-phenylene)bis(3-aryl-4,5dihydro-1H-pyrazole-5,1-diyl))diethanone, and bis-N-phenylpyrazole (7a-e), 1,3-bis(1-phenyl-3-(aryl)4,5-dihydro-1H-pyrazol-5-yl)benzene, derivatives were obtained by addition of hydrazine hydrate and/or phenyl hydrazine to bis-chalcone derivatives (3a-e) in acetic acid and/or acetic acid/ethanol at reflux conditions for 4 and 8 hours, respectively. The structures of obtained bis-N-acetylpyrazole (5a-e) and bis-N-phenylpyrazole (7a-e) derivatives were characterized using the spectroscopic methods (NMR, IR) and elemental analysis, and comparison with literature data. ${ }^{27}$ All spectral data are in good agreement with the proposed structures.

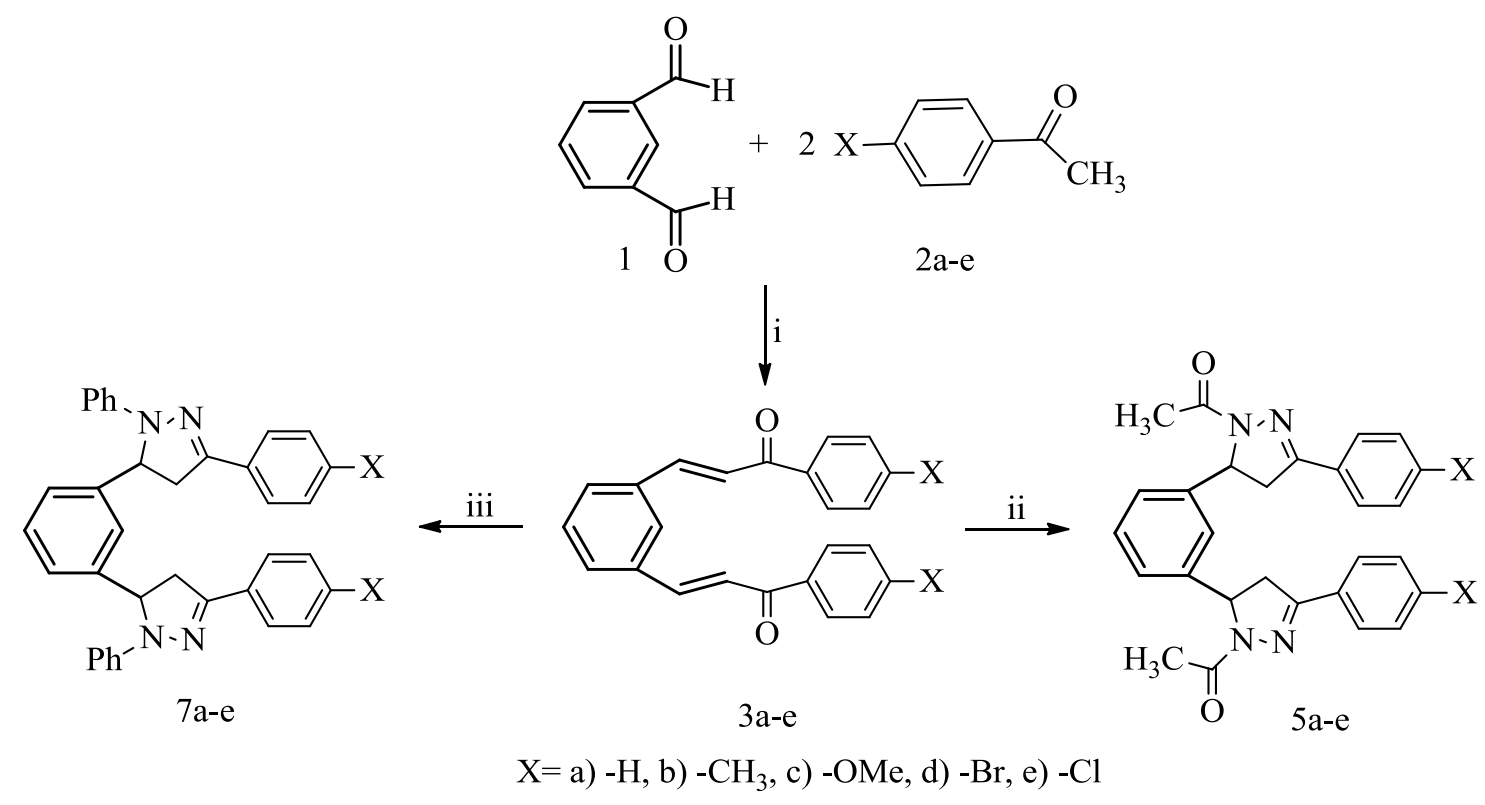

Reagents and conditions: i) $\mathrm{NaOH}$, EtOH, r.t. 4 h.; ii) $\mathrm{H}_{2} \mathrm{NNH}_{2} \cdot \mathrm{H}_{2} \mathrm{O}$ (4), $\mathrm{CH}_{3} \mathrm{COOH}$, ref. 4 h.;

iii) $\mathrm{PhNHNH}_{2}, \mathrm{AcOH} / \mathrm{EtOH}$, ref. $8 \mathrm{~h}$.

Scheme 1. Synthetic pathway of compounds 3(a-e), 5(a-e) and 7(a-e) 


\subsection{Biological assays}

\subsubsection{Antimicrobial activity results}

The synthesized compounds (5a-e) were tested for their antibacterial activity against five different types of human pathogenic bacterial strains, Enterococcus faecalis (ATCC® 29212), Staphylococcus aureus (ATCC®29213) which are Gram-positive bacteria, Escherichia coli (ATCC $\left.{ }^{\circledR} 25922\right)$, Pseudomonas aeruginosa (ATCC®27853), which are Gram-negative bacteria and Candida albicans (ATCC®1213) which is yeast. Gentamycin and Fluconazole were used as standard and DMSO was used as negative control in the tests. The results were given as inhibition zone (mm) compared with standards (Table 2).

Table 1. Synthesized compounds 3(a-e), 5(a-e) and 7(a-e)

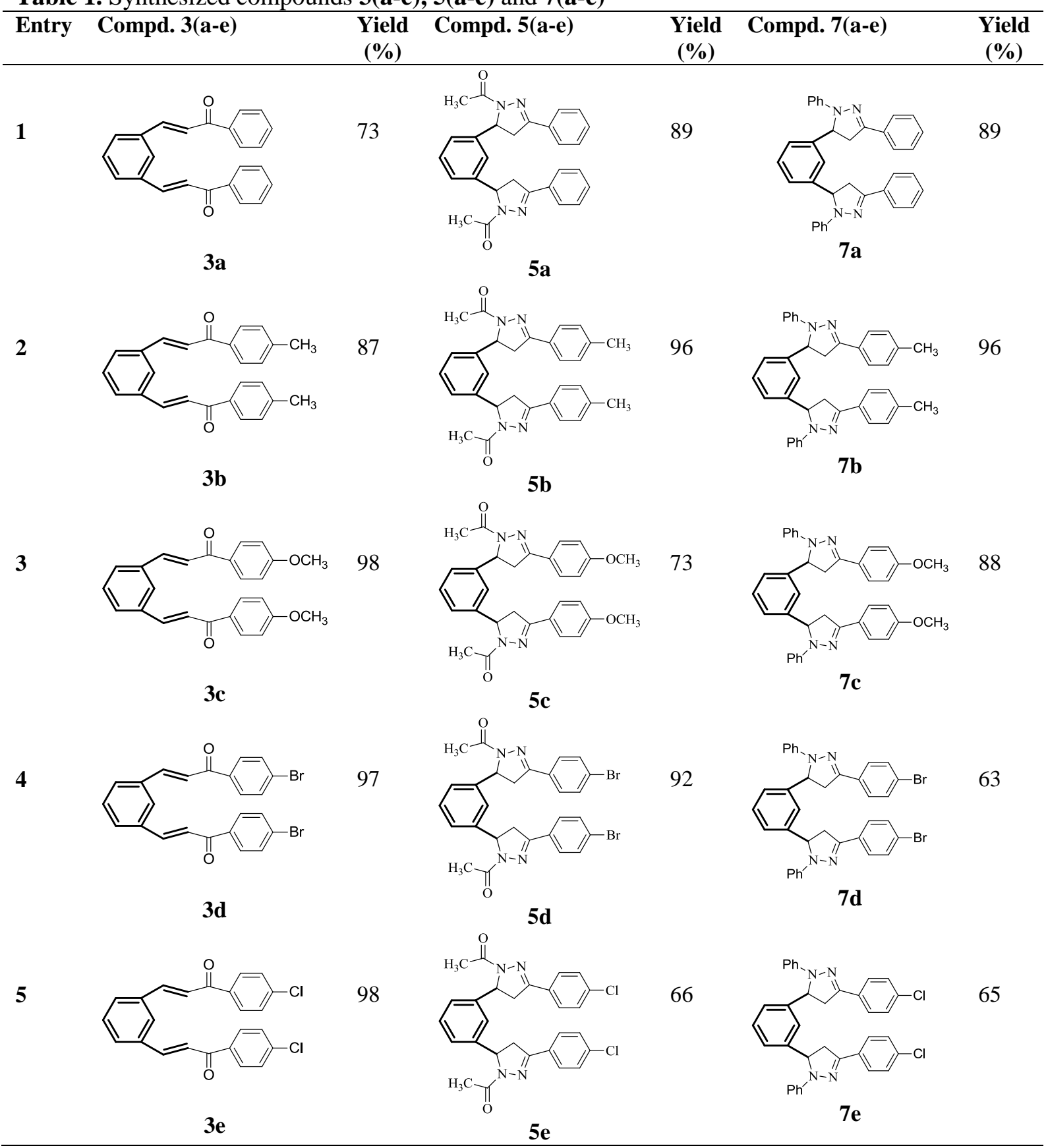


According to Table 2, compounds $\mathbf{5 b}$ showed low activity with $\mathbf{3} \mathbf{m m}$ of inhibition zone and $\mathbf{5 c}$ showed good activity with $6.5 \mathrm{~mm}$ of inhibition zone against E. faecalis compared to standard (Gentamycin $=10.5 \mathrm{~mm}$ ), and the others compounds were inactive. Also, compounds $\mathbf{5 b}$ and $\mathbf{5 c}$ displayed good activity with $5.5 \mathrm{~mm}$ and $6 \mathrm{~mm}$ of inhibition zone against $S$. aereus compared to standard (Gentamycin $=8.25 \mathrm{~mm}$ ), and the others were inactive. Addition, compounds $\mathbf{5 b}, \mathbf{5 c}$ and $\mathbf{5 e}$ demonstrated low activity with $1.5 \mathrm{~mm}, 2.5 \mathrm{~mm}$ and $3.75 \mathrm{~mm}$ of inhibition zone, respectively, against E. coli 111 compared to standard (Gentamycin $=11.5 \mathrm{~mm}$ ), and the others were inactive. All compounds 5a-e exhibited low activity with $0.75-4.5 \mathrm{~mm}$ of inhibition zone against $P$. aeroginosa compared to standard (Gentamycin $=9.75 \mathrm{~mm})$. The most active compounds were $\mathbf{5 d}(10.5 \mathrm{~mm})$ and 5e $(8.0 \mathrm{~mm})$, and they were showed almost the same activity with standard Fluconazole $(10.75 \mathrm{~mm})$ against $C$. albicans. According to these results, further researches can be performed for compounds $\mathbf{5 d}$ and $\mathbf{5 e}$ as potential antibacterial agents against $C$. albicans.

Table 2. In vitro antimicrobial activites of compounds 5a-e

\begin{tabular}{cccccc}
\hline & \multicolumn{5}{c}{ Antimicrobial activities (inhibition zone (mm)) } \\
\cline { 2 - 6 } Comp. & E. faecalis & S. aereus & E. coli & \multicolumn{2}{l}{ P. } \\
& & & - & aeroginosa & C. albicans \\
\hline $\mathbf{5 a}$ & - & - & - & $4.5 \pm 1.0$ & $1.5 \pm 0.5$ \\
$\mathbf{5 b}$ & $3.0 \pm 1.5$ & $5.5 \pm 0.5$ & $1.50 \pm 0.5$ & $1.0 \pm 0.5$ & $1.0 \pm 0.25$ \\
$\mathbf{5 c}$ & $6.5 \pm 2.0$ & $6.0 \pm 1.00$ & $2.5 \pm 1.0$ & $0.75 \pm 0.25$ & $2.0 \pm 1.0$ \\
$\mathbf{5 d}$ & - & - & - & $1.0 \pm 0.5$ & $10.5 \pm 2.0$ \\
$\mathbf{5 e}$ & - & - & $3.75 \pm 1.5$ & $1.5 \pm 0.5$ & $8.0 \pm 1.75$ \\
Stand. & $10.25 \pm 2.00$ & $8.25 \pm 1.70$ & $11.5 \pm 2.50$ & $9.75 \pm 2.25$ & - \\
Stand. & - & - & - & - & $10.75 \pm 1.25$ \\
DMSO & - & - & - & - & - \\
\hline
\end{tabular}

Standards: ${ }^{\mathrm{a}}$ Gentamycin; ${ }^{\mathrm{b}}$ Fluconazole; -: Inactive.

\subsubsection{Antioxidant Activity Test}

The synthesized compounds were also tested in terms of Trolox equivalent and the test results show that all of the compounds have antioxidant capacity (Table 3 ).

Table 3. Antioxidant activities of compounds 5(a-e)

\begin{tabular}{llllll}
\hline & $\mathbf{5 a}$ & $\mathbf{5 b}$ & $\mathbf{5 c}$ & $\mathbf{5 d}$ & $\mathbf{5 e}$ \\
\hline Antioxidant activity (mmol Trolox eq./L.): & 2.3 & 3.1 & 2.6 & 0.8 & 3.9 \\
\hline
\end{tabular}

\section{Conclusion}

Conclusion, The 1,3-Phenylene-bis-N-acetylpyrazoles (5a-e) and 1,3-Phenylene-bis-Nphenylpyrazoles (6a-e) were synthesized by addition of hydrazine hydrate and/or phenylhydrazine to 1,3-phenylene-bis-chalcone derivatives (3a-e), respectively in high yields. Addition, the in vitro antibacterial activities of compounds (5a-e) was tested against the five human pathogenic bacteria. Among the compounds 5a-e, compounds 5d and 5e showed the same activity with standard (Fluconazole) against $C$. albinas.

\section{Acknowledgements}

The authors are indebted to the TUBITAK (2209-A Domestic Research Project Support Program for University Students) for financial supports. 


\section{References}

[1] Damljanovic, I.; Vukicevic, M.; Radulovic, N.; Palic, R.; Ellmerer, E.; Ratkovic, Z.; Joksovic, M. D.; Vukicevic, R. D. Synthesis and antimicrobial activity of some new pyrazole derivatives containing a ferrocene unit. Bioorg. Med. Chem. Lett. 2009, 19(4), 1093-1096.

[2] Dardari, Z.; Lemrani, M.; Sebban, A.; Bahloul, A.; Hassar, M.; Kitane, S.; Berrada, M.; Boudouma, M. Antileishmanial and antibacterial activity of a new pyrazole derivative designated 4-[2-(1-(Ethylamino)-2methyl- propyl)phenyl]-3-(4- methyphenyl) -1- phenylpyrazole. Arch. Pharm. 2006, 339(6), 291-298.

[3] Ahasan, N. B.; Rabiul Islam, M. D. Cytotoxicity study of pyrazole derivatives. Bangladesh J. Pharmacol. 2007, 2, 81-87.

[4] Sahu, S. K.; Banerjee, M.; Samantray, A.; Behera, C.; Azam, M. A. Synthesis, analgesic, anti-inflammatory and antimicrobial activities of some novel pyrazoline derivatives. Tropical J. Pharma. Res. 2008, 7(2), 961968.

[5] Mogilaiah, K.; Chowdary, D. S.; Rao, R. B. Synthesis and antibacterial activity of pyrazole and 1,3,4oxadiazole derivatives of 2-phenyl-1,8-naphthyridine. Indian J. Chem. 2001, 40B, 43.

[6] Insuasty, B.; Montoya, A.; Becerra, D.; Quiroga, J.; Abonia, R.; Robledo, S.; Darío Velez, I.; Upegui, Y.;Nogueras, M.; Cobo, J. Synthesis of novel analogs of 2-pyrazoline obtained from [(7-chloroquinolin4-yl)amino]chalcones and hydrazine as potential antitumor and antimalarial agents. Eur. J. Med. Chem. 2013, 67, 252.

[7] Ramamurthy, V.; Venkatesan, K. Photochemical reactions of organic crystals. Chem. Rev., 1987, 87, 433481.

[8] Desiraju, G. R.; Goud, B. S. Reactivity of Solids Past, Present and Future, edited by oldyrev V V (Blackwell Science, London), 1994, 233.

[9] Abd El-Wahab, A.H.F.; Al-Fifi, Z.I.A.; Bedair, A.H.; Ali, F.M.; Halawa, A.H.A.; El- Agrody, A.M. Synthesis, reactions and biological evaluation of some new naphtho[2,1- b]furan derivatives bearing a pyrazole nucleus. Molecules. 2011, 16, 307-318.

[10] Ouyang, G.P.; Cai, X.J.; Chen, Z.; Song, B.A.; Bhadury, P.S.; Yang, S.; Jin, L.H.; Xue, W.; Hu, D.Y.; Zeng, S. Synthesis and antiviral activities of pyrazole derivatives containing oxime ethers moiety. J. Agric Food Chem. 2008, 56, 10160-10167.

[11] Saad, H.A.; Osman, N.A.; Moustafa, A.H. Synthesis and analgesic activity of some new pyrazoles and triazoles bearing a 6,8-dibromo-2-methylquinazoline moiety. Molecules. 2011, 16, 10187-10201.

[12] Liu, X.H.; Cui, P.; Song, B.A.; Bhadury, P.S.; Zhu, H.L.; Wang, S.F. Synthesis, structure and antibacterial activity of novel 1-(5-substituted-3-substituted-4,5-dihydropyrazol-1-yl)ethanone oxime ester derivatives. Bioorgan. Med. Chem. 2008,16, 4075-4082.

[13] Ouyang, G.; Chen, Z.; Cai, X.J.; Song, B.A.; Bhadury, P.S.; Yang, S.; Jin, L.H.; Xue,W.; Hu, D.Y.; Zeng, S. Synthesis and antiviral activity of novel pyrazole derivatives containing oxime esters group. Bioorgan. Med. Chem. 2008, 16, 9699-9707.

[14] Abdel-Hafez, E.M.N.; Rahma, G.E.A.A.; Aziz, M.A.; Radwan, M.F.; Farag, H.H. Design, synthesis and biological investigation of certain pyrazole-3-carboxylic acid erivatives as novel carriers for nitric oxide. Bioorgan. Med. Chem. 2009, 17, 3829-3837.

[15] Park, H.J.; Lee, K.; Park, S.J.; Ahn, B.; Lee, J.C.; Cho, H.Y.; Lee, K.I. Identification of antitumor activity of pyrazole oxime ethers. Bioorgan. Med. Chem. Lett. 2005, 15, 3307-3312.

[16] Ouyang, G.; Cai, X.J.; Chen, Z.; Song, B.A.; Bhadury, P.S.; Yang, S.; Jin, L.H.; Xue, W.; Hu, D.Y.; Zeng, S. Synthesis and antiviral activities of pyrazole derivatives containing an oxime moiety. J. Agric. Food Chem. 2008, 56, 101601-102607.

[17] Dai, H.; Li, Y.Q.; Du, D.; Qin, X.; Zhang, X.; Yu, H.B.; Fang, J.X. Synthesis and biological activities of novel pyrazole oxime derivatives containing a 2-chloro-5- thiazolyl moiety. Food Chem. 2008, 56, 1080510810.

[18] Pinto, D. C. G. A.; Silva, A. M. S.; Cavaleiro, J. A. S.; Elguero, J. New bis(chalcones) and their transformation into bis(pyrazoline) and bis(pyrazole) derivatives. Eur. J. Org. Chem. 2003, 747-755.

[19] Gürdere, M. B.; Özbek, O.; Ceylan, M. Aluminum chloride-catalyzed C-alkylation of pyrrole and indole with chalcone and bis-chalcone derivatives. Synth. Commun. 2016,46, 322-331.

[20] Budak, Y. Potassium tert-butoxide catalyzed synthesis and characterization of novel -aryl-3-(phenylthio)-1(thiophen-3-yl)- propan-1-one Derivatives. Chinese. J. Chem., 2012, 30, 341-344. 
[21] Ceylan, M.; Gürdere, M. B.; Gezegen, H.; Budak, Y. Potassium-tertiary butoxide-assisted addition of thioglicolic acid to chalcone derivatives under solvent-free conditions. Synth. Commun. 2010, 40, 2598-2606.

[22] Voskiene, A.; Mickevicius, V.; Mikulskiene, G. Synthesis and structural characterization of products condensation 4-carboxy-1-(4-styrylcarbonylphenyl)-2-pyrrolidinones with hydrazines. Arkıvoc. 2007, 15, 303-314.

[23] Yerli, G.; Gezegen, H.; Ceylan, M. Iodine-catalyzed addition of 2-mercaptoethanol to chalcone derivatives: Synthesis of the novel $\beta$-mercapto carbonyl compounds. Org. Commun. 2012, 5:2, 70-76

[24] Wang, P.; Onozawa-Komatsuzaki, N.; Himeda, Y.; Sugihara, H.; Arakawa, H.;Kasuga, K. 3-(2-yridyl)2-pyrazoline derivatives: novel fluorescent probes for $\mathrm{Zn}^{2+}$ ion. Tetrahedron Lett. 2001, 42, 9199-9201.

[25] El-Rayyes, N.; Ai-Johary, A. A. Heterocycles. 7. Synthesis of new pyrazolines. J Chem. Eng. Data. 1985, 30, 500-502.

[26] Zhang, X. H.; Lai, W.Y.; Gao, Z. Q.; Wong, T.C.; Lee, C. S.; Kwong, H. L.; Lee, S. T.; Wu, S. K. Photoluminescence and electroluminescence of pyrazoline monomers and Dimmers. Phys. Lett Chem. 2000, 320, 77-80.

[27] Gürdere, M. B.; Gümüş, O.; Sahin Yaglioglu, A.; Budak, Y.; Ceylan, M. Synthesis and anticancer activities of 1,4-Phenylene-bis-N-acetyl- and N-phenylprazoline derivatives. Res. Chem. Intermed. 2017, 43, 12771289.

[28] Ceylan, M.; Gurdere, M.B.; Karaman, I.; Gezegen, H. The synthesis and screening of the antimicrobial activity of some novel 3-(furan-2-yl)-1-(aryl)-3-(phenylthio) propan-1-one derivatives. Med. Chem. Res. 2011, 20, 109-115.

[29] Karaman, İ.; Gezegen, H.; Gürdere, M.B.; Dingil, A.; Ceylan, M. Screening of biological activities of a series of chalcone derivatives against human pathogenic microorganisms. Chem. Biodiver. 2010, 7, 400-408.

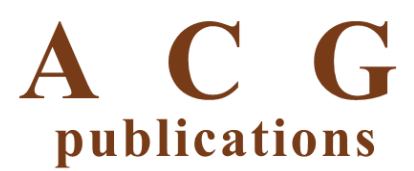

(C) 2017 ACG Publications. 